\title{
Detection of diffuse interstellar bands in M 31
}

\author{
M. A. Cordiner ${ }^{1}$, N. L. J. Cox ${ }^{2}$, C. Trundle ${ }^{1}$, C. J. Evans ${ }^{3}$, I. Hunter ${ }^{1}$, N. Przybilla ${ }^{4}$, F. Bresolin ${ }^{5}$, and F. Salama ${ }^{6}$ \\ 1 Astrophysics Research Centre, School of Mathematics and Physics, Queen's University, Belfast, BT7 1NN, UK \\ e-mail: m.cordiner@qub.ac.uk \\ 2 Herschel Science Centre, European Space Astronomy Centre, ESA, PO Box 78, 28691 Villanueva de la Cañada, Madrid, Spain \\ 3 UK ATC, Royal Observatory Edinburgh, Blackford Hill, Edinburgh, EH9 3HJ, UK \\ 4 Dr. Remeis-Sternwarte Bamberg, Sternwartstr. 7, 96049 Bamberg, Germany \\ 5 Institute for Astronomy of the University of Hawaii, 2680 Woodlawn Drive, 96822 Honolulu, HI, USA \\ 6 Space Science Division, NASA Ames Research Center, Mail Stop 245-6, Moffett Field, California 94035, USA
}

Received 21 December 2007 / Accepted 22 January 2008

\section{ABSTRACT}

\begin{abstract}
Aims. We investigate the diffuse interstellar band (DIB) spectrum in the interstellar medium of M 31 .
Methods. The DEIMOS spectrograph of the W. M. Keck observatory was used to make optical spectroscopic observations of two supergiant stars, MAG 63885 and MAG 70817, in the vicinity of the OB78 association in M 31 where the metallicity is approximately equal to solar.

Results. The $\lambda \lambda 5780,5797,6203,6283$ and 6613 DIBs are detected in both sightlines at velocities matching the M 31 interstellar Na I absorption. The spectra are classified and interstellar reddenings are derived for both stars. Diffuse interstellar band (DIB) equivalent widths and radial velocities are presented.

Conclusions. The spectrum of DIBs observed in M31 towards MAG63885 is found to be similar to that observed in the Milky Way. Towards MAG 70817 the DIB equivalent widths per unit reddening are about three times the Galactic average. Compared to observations elsewhere in the Universe, relative to reddening the M 31 ISM in the vicinity of OB78 is apparently a highly favourable environment for the formation of DIB carriers.
\end{abstract}

Key words. astrochemistry - galaxies: Local Group - galaxies: ISM - ISM: lines and bands - ISM: clouds - ISM: dust, extinction

\section{Introduction}

Today, more than 300 diffuse interstellar bands (DIBs) are known but the carriers have remained unidentified since their discovery almost 100 years ago. It is debated whether the DIB carriers arise from the dust, the gas, or the large-molecule component of the interstellar medium (see the review by Sarre 2006). The substructure present in many of the DIB profiles indicates (Sarre et al. 1995; Ehrenfreund \& Foing 1996) that they are caused by large gas-phase molecules. The interaction between gas-phase species (atoms and molecules), UV radiation and dust grains may be crucial in the formation of large ( $\gtrsim 50$ atom) molecules in space which are likely candidates for the carriers of the DIBs (see e.g. Salama et al. 1996; Ruiterkamp et al. 2005).

In interstellar environments distinct from those found in the Milky Way (MW), previous research on the relationships between atoms, molecules, dust and DIBs has focused on the Large and Small Magellanic Clouds (e.g. Ehrenfreund et al. 2002; Cox et al. 2006, 2007; Welty et al. 2006). The behaviour of the DIB carriers was analysed with respect to the higher gas-todust ratios, lower metallicities, lower $R_{V}$ and stronger interstellar radiation fields of these environments. Beyond the Magellanic Clouds, studies are sparse, confined to sightlines probed by sufficiently bright supernovae (e.g. Rich 1987; Sollerman et al. 2005) or background quasars (e.g. York et al. 2006).

M31 presents the opportunity to study the effects on the DIB carriers of the unique chemical and physical conditions found in this Local Group galaxy. This letter presents spectra of two supergiant stars in M 31 and we report the first unambiguous detection of DIBs in M31. The observed DIB properties are discussed in relation to those in other galaxies and the interstellar conditions of M31.

\section{Observations}

Seventy-two bright stars in and around the M 31 OB 78 association (van den Bergh 1964) were observed in November 2003 using the Keck DEIMOS spectrograph. Two angles of the $1200 \mathrm{G}$ grating were used to cover the region from approximately 3500 to $9000 \AA$ with a resolving power $R=3300$. The total exposure time was $2.25 \mathrm{~h}$ in the blue region and $1.5 \mathrm{~h}$ in the red, during which the seeing was $0.5-0.8^{\prime \prime 1}$. Wavelength calibration accuracy was confirmed using the $\mathrm{Na} \mathrm{D}$ sky emission lines whose central wavelengths were found to be accurate to within $\pm 5 \mathrm{~km} \mathrm{~s}^{-1}$. Reduced spectra were Doppler-corrected to the LSR frame. Table 1 shows the co-ordinates and photometry of MAG 63885 and MAG 70817. These targets were selected for analysis in this letter due to their prominent DIBs. The rest of the DEIMOS data will be presented in a future article (Cordiner et al. 2008).

\footnotetext{
${ }^{1}$ Initially the red and blue spectra were both reduced using the spec $2 \mathrm{~d}$ pipeline. However, the wavelength scale of the blue spectra ( 3500-6000 $\AA$ ) was found to be erroneous so these were re-reduced manually in IRAF using the twodspec routines.
} 
Table 1. Observed M 31 stars. Target numbers from Magnier et al. (1992). Co-ordinates and photometry from Massey et al. (2006). Derived stellar spectral classifications and radial velocities $\left(v_{*}\right)$ are shown. M $31 E_{B-V}$ values were calculated using intrinsic colours from Johnson (1966) and have been corrected for Galactic foreground reddening (see Sect. 3.4).

\begin{tabular}{lcccccccc}
\hline \hline $\begin{array}{l}\text { Target } \\
\text { MAG\# }\end{array}$ & $\begin{array}{c}\text { RA } \\
\text { J2000 }\end{array}$ & $\begin{array}{c}\text { Dec } \\
\text { J2000 }\end{array}$ & $\begin{array}{c}V \\
(\mathrm{mag})\end{array}$ & $\begin{array}{c}B-V \\
(\mathrm{mag})\end{array}$ & Sp. type & $\begin{array}{c}E_{B-V} \\
(\mathrm{mag})\end{array}$ & $\begin{array}{c}v_{*} \\
\left(\mathrm{~km} \mathrm{~s}^{-1}\right)\end{array}$ & $\begin{array}{c}S / N \\
@ 5800 \AA\end{array}$ \\
\hline 63885 & $00: 40: 30.47$ & $+40: 35: 03.8$ & $16.380 \pm 0.003$ & $0.326 \pm 0.003$ & B9 Ia & $0.28 \pm 0.04$ & -544 & 150 \\
70817 & $00: 40: 35.46$ & $+40: 36: 44.6$ & $18.282 \pm 0.003$ & $0.431 \pm 0.003$ & F2 I & $0.12 \pm 0.04$ & -543 & 80 \\
\hline
\end{tabular}

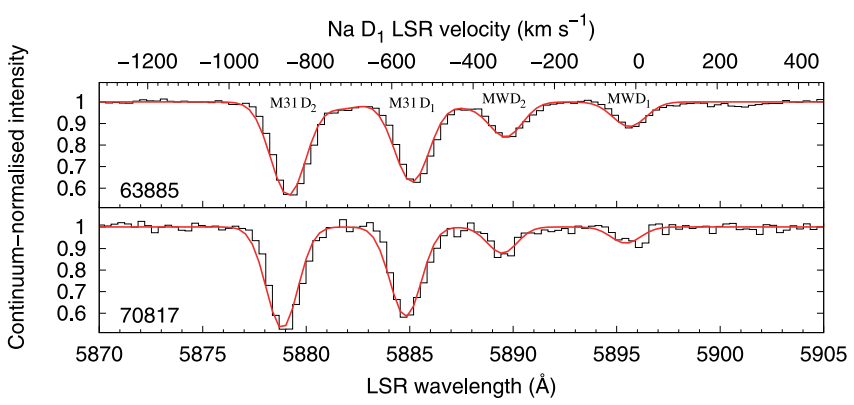

Fig. 1. Observed continuum-normalised Na I spectra (histograms) with best-fitting models overlaid (red curves). Velocity scale at the top is relative to the $\mathrm{Na} \mathrm{D}_{1}$ rest wavelength. The M31 and Galactic (MW) absorption components are labelled.

\section{Analysis and results}

\subsection{Interstellar sodium $D$}

The observed Na D lines (see Fig. 1) show two clearly separated absorption components due to interstellar gas at velocities consistent with the Galactic and M 31 ISM (around radial velocities of 0 and $-550 \mathrm{~km} \mathrm{~s}^{-1}$ respectively). The spectra were modelled using VAPID (Howarth et al. 2002) and each absorption component was accurately reproduced with a Gaussian interstellar cloud model. The fits are plotted in Fig. 1 and the mean radial velocities and equivalent widths (EWs) of the M $31 \mathrm{D}_{1}$ absorption components are given in Table 2.

\subsection{Diffuse interstellar bands}

We searched the spectra for all DIBs with central depths greater than 0.05 in Fig. $6^{2}$ of the DIB survey by Jenniskens \& Desert (1994). Scaled to the reddening of our targets, this central depth limit corresponds to $\sim 1 \sigma$ of the spectral Poisson noise. The $\lambda \lambda 5780,5797,6203,6283$ and 6613 DIBs were detected at M 31 velocities in the spectra of MAG 63885 and MAG 70817. $\lambda 6196$ was tentatively detected towards MAG70817. The $\lambda \lambda 4501$, 4726, 5705, 5849, 6269, 6376, 6379, 6445, 6532, 6660, 6993 and 8026 DIBs were too weak to be detected. Telluric absorption line contamination prevented analysis of the $\lambda \lambda 6886,6919$, 7224 and 7334 DIBs.

To simultaneously measure the DIB radial velocities and equivalent widths, Galactic DIB templates were fitted to the observed spectra using a non-linear least-squares algorithm. The Galactic templates were derived from high resolution, high $\mathrm{S} / \mathrm{N}$ spectra of $\beta^{1} \mathrm{Sco}$ (see Cordiner 2006). These were shifted to the interstellar rest frame and convolved with a Gaussian interstellar cloud model then with the instrumental spectral PSF and rebinned to the wavelength scale of the DEIMOS spectra. This technique minimises statistical errors in the measurement

\footnotetext{
${ }^{2}$ A synthetic average of their observed DIB spectra (normalised to $E_{B-V}=1.28$ ).
}

Table 2. Summary of best-fitting DIB equivalent widths $(E W / \mathrm{m} \AA)$ and LSR radial velocities $\left(v / \mathrm{km} \mathrm{s}^{-1}\right)$ for MAG 63885 and MAG 70817. $f$ denotes values held fixed during fitting. Statistical (68th percentile) DIB velocity errors are less than about $\pm 10 \mathrm{~km} \mathrm{~s}^{-1}$. The M $31 \mathrm{Na}$ I radial velocities and $\mathrm{D}_{1}$ line $E W \mathrm{~s}$ are also shown, including an estimated contribution due to stellar photospheric NaI in square brackets. $E W \mathrm{~s}$ for $\beta^{1} \operatorname{Sco}\left(E_{B-V}=0.22\right)$ and Galactic mean $E W / E_{B-V}$ data (normalised to $E_{B-V}=0.14$ ) are also given (data from Herbig 1993; Thorburn et al. 2003; Megier et al. 2005; and Cordiner 2006).

\begin{tabular}{lllllll}
\hline \hline & \multicolumn{2}{c}{ MAG 63885 } & \multicolumn{2}{c}{ MAG 70817 } & $\beta^{1}$ Sco & \multirow{2}{*}{ MW Avg. } \\
& \multirow{2}{*}{$E W$} & $v$ & $E W$ & $v$ & $E W$ & $E W$ \\
\hline 5780 & $190_{-16}^{+26}$ & -539 & $271_{-26}^{+17}$ & -559 & 142 & 75 \\
5797 & $44_{-5}^{+8}$ & $-550^{f}$ & $58_{-16}^{+32^{\prime}}$ & $-565^{f}$ & 17 & 22 \\
6196 & $3_{-3}^{+9^{2}}$ & $-550^{f}$ & $29_{-9}^{+19}$ & $-565^{f}$ & 12 & 10 \\
6203 & $94_{-16}^{+21}$ & $-550^{f}$ & $121_{-13}^{+15}$ & $-565^{f}$ & 57 & 23 \\
6283 & $260_{-11}^{+11}$ & -575 & $541_{-21}^{+21}$ & -558 & 330 & 159 \\
6613 & $63_{-8}^{+7}$ & -564 & $74_{-12}^{+11}$ & -548 & 43 & 31 \\
$\mathrm{NaD} \mathrm{D}_{1}$ & $736[70]$ & -550 & $846[300]$ & -565 & 147 & \\
\hline
\end{tabular}

of the equivalent widths and velocities of weak DIBs but assumes that the intrinsic M31 DIB profiles and rest wavelengths closely match those of $\beta^{1}$ Sco. If this is not the case, unknown systematic errors could occur, but we find no evidence to suggest that M 31 DIB profiles differ at all from those of $\beta^{1}$ Sco. Observed DIB spectra and fitted profiles are shown in Fig. 2.

For the $\lambda \lambda 5780,6283$ and 6196 DIBs the radial velocities and equivalent widths were allowed to vary in the fits. A telluric absorption component (derived from a high-resolution spectrum of the unreddened fast-rotator $\alpha$ Gru (see Cordiner 2006), degraded to $R=3300$ ), was also included for $\lambda 6283$ to account for the prominent band-head of atmospheric $\mathrm{O}_{2}$ between 6278 and $6286 \AA^{3}$. For the $\lambda \lambda 5797,6196$ and 6203 DIBs only the equivalent widths were allowed to vary while the DIB radial velocities were fixed at the M31 Na I radial velocities. In all cases, there is excellent agreement between the fitted and observed DIB profiles. DIB radial velocities and $E W \mathrm{~s}$ are shown in Table 2. The radial velocities of all measured DIBs closely match the Na I radial velocities, consistent with previous high-resolution studies of the Galactic and extragalactic ISM (such as those by Sollerman et al. 2005, and Cox et al. 2006, 2007).

Statistical equivalent width and DIB velocity error estimates were derived by replicating the spectra 100 times with randomly generated Poisson noise added to each. The DIB model parameters were refitted for each replication and individual parameter errors were obtained from the \pm 68 th percentiles of the resulting parameter ranges.

We also found evidence for a broad absorption feature towards MAG 63885 centred near $4430 \AA$, identified as a blend of M 31 and Milky Way 24428 DIBs. This feature was modelled as a sum of two Lorentzian components with FWHM $17.25 \AA$

\footnotetext{
3 Our DEIMOS observations contained no telluric standards by which to perform a conventional telluric division.
} 

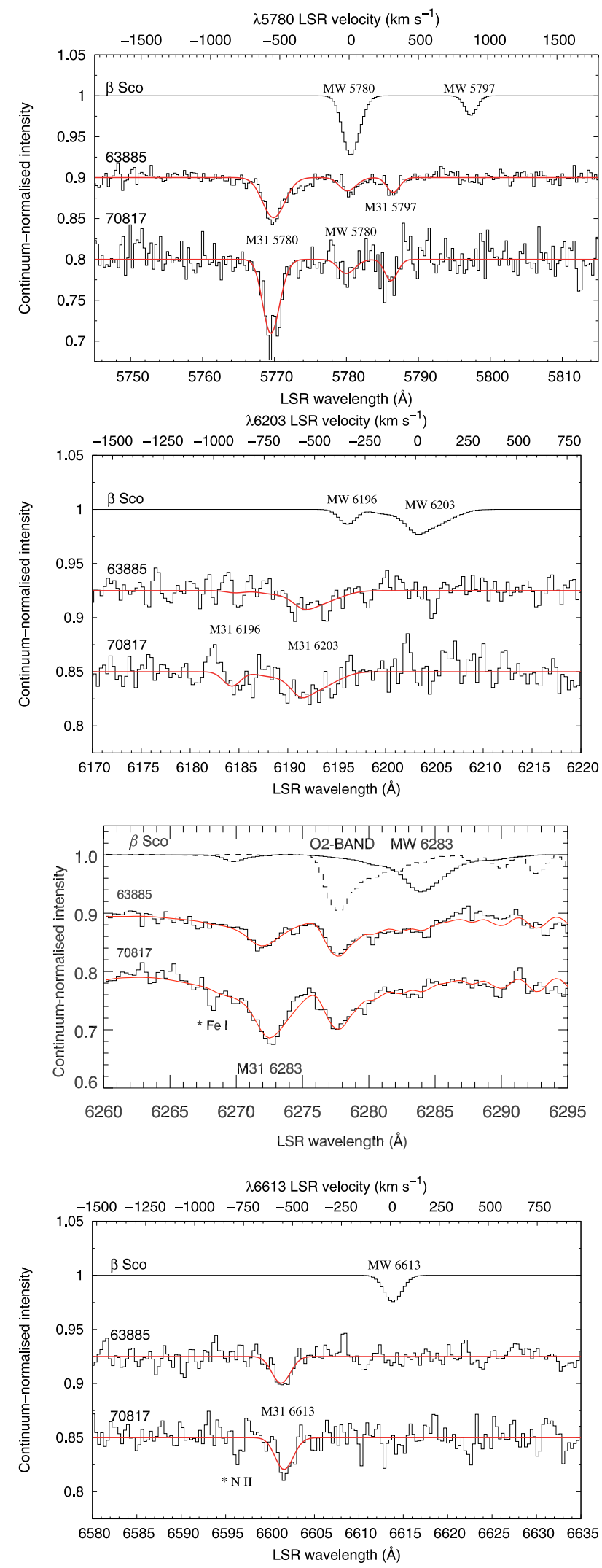

Fig. 2. Continuum-normalised spectra of the observed DIBs (black histograms). Galactic (MW) and M31 DIBs are labelled. Prominent stellar lines are marked with asterisks. Fitted model DIB profiles are overlaid (red curves). Velocity scales at the top are relative to the Galactic DIB rest wavelengths published by Galazutdinov et al. (2000). $\beta^{1}$ Sco Galactic reference DIB templates (plotted with arbitrary intensity scaling factors), are shown at the top of each figure, with additional telluric $\mathrm{O}_{2}$-band spectrum (dashed line) for the $\lambda 6283$ region. (see Snow 2002) at $v=0$ and $v=-550 \mathrm{~km} \mathrm{~s}^{-1}$. The best-fitting model yields $1057 \mathrm{~m} \AA$ for the M 31 component and $236 \mathrm{~m} \AA$ for the MW foreground component. However, this result is uncertain due to stellar line contamination and the relatively poor signal-to-noise in this spectral region.

\subsection{Stellar spectral types}

Spectral types and stellar radial velocities for the target stars are presented in Table 1. Analysis of luminous B-type supergiants in M31 finds chemical abundances comparable to those in the solar neighbourhood (e.g. Trundle et al. 2002), so Galactic standards are appropriate. The spectra were classified with reference to the Galactic standards from Evans \& Howarth (2003), with luminosity classes assigned on the basis of the $\mathrm{H} \gamma$ equivalent widths (Evans et al. 2004).

Stellar radial velocities are the mean results from manual measurements of the cores of the $\mathrm{H} \alpha$ Balmer line and the Paschen lines. Statistical errors on the measured radial velocities are less than $\pm 5 \mathrm{~km} \mathrm{~s}^{-1}$.

We searched Galactic stellar spectra of the same spectral types as our targets for the presence of lines overlapping the detected DIBs. No significant contamination of the $\lambda \lambda 6196,6203$, 6283 or 6613 DIBs is expected. The $\lambda \lambda 5780$ and 5797 may suffer contamination of up to about 5 and $2 \mathrm{m \AA}$ respectively as a result of overlapping lines of Fe I.

\subsection{Foreground gas and dust}

LAB H I data (Kalberla et al. 2005) for the nearest survey point in the direction of our sightlines shows $N(\mathrm{HI})=5.91 \times$ $10^{20} \mathrm{~cm}^{-2}$ over the velocity range of Galactic gas (from -300 to $100 \mathrm{~km} \mathrm{~s}^{-1}$ ). Equation (7) of Burstein \& Heiles (1978) is used to calculate the foreground reddening for lines of sight at latitudes away from the Galactic plane which yields $E_{B-V}=0.06$ mag towards our targets, identical to the value given by the foreground dust map of Schlegel et al. (1998).

The Galactic foreground Na I column densities measured towards MAG 63885 and MAG 70817 are $3.7 \times 10^{12}$ and $1.9 \times$ $10^{12} \mathrm{~cm}^{-2}$ respectively, which correspond to reddenings of 0.12 and 0.08 mag (Hobbs 1974). However, there may be contamination of the Galactic Na I profiles near $v=0$ due to sky-line subtraction residuals. Using a foreground reddening of 0.06 , corrected $\mathrm{M} 31 E_{B-V}$ values are given in Table 1.

\section{Discussion}

DIB equivalent widths in the Milky Way correlate with $E_{B-V}$ (Herbig 1995). Given the signal-to-noise and the limited reddening of our target spectra, only the strongest known Galactic DIBs were detected in M 31. Other M 31 DIBs (including those listed in Sect. 3.2) are expected in our spectra around or below the limit of detectability.

The DIB equivalent widths observed in M31 toward MAG63885 are consistent with those typically observed in Galactic sightlines with the same reddening, as highlighted by comparison with the similarly-reddened $\beta^{1}$ Sco (see Table 2). Towards MAG 70817 the observed DIBs are stronger per unit $E_{B-V}$ by a factor of about 2-5 compared with Galactic-mean data. Figure 3 shows that the $\lambda 5780 \mathrm{EW} / \mathrm{E}_{B-V}$ for MAG 63885 is among the larger values observed elsewhere in the Universe and for MAG $70817 \lambda 5780 \mathrm{EW} / E_{B-V}$ is significantly greater than any other sightline plotted. 


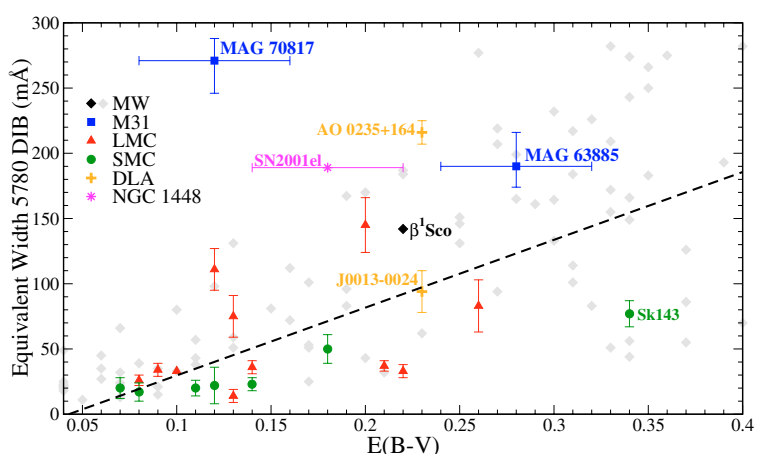

Fig. 3. $\lambda 5780$ equivalent widths $v s$. reddening for different galaxies. Data for the LMC and SMC (Welty et al. 2006), DLAs (York et al. 2006; Ellison et al. 2008) and NGC 1448 (Sollerman et al. 2005) are shown. The dashed line shows a linear fit to Galactic data (from Herbig 1993; Thorburn et al. 2003; Megier et al. 2005; Cordiner 2006).

Detailed information is sparse, but M 31 appears be similar to the Milky Way in terms of the average interstellar gas-to-dust ratio (Nedialkov et al. 2000; Bresolin et al. 2002) and the metallicity in the vicinity of our targets (Trundle et al. 2002). There is, however, evidence for a difference in the properties of the interstellar dust grains and the interstellar UV radiation field. A different star formation history and low rate of star formation (1/10th of MW; Walterbos \& Braun 1994) has been measured in M 31, and the surface radiation flux was found to be poor in UV (Cesarsky et al. 1998; Pagani et al. 1999). The fact that strong DIBs are observed under these conditions may be in contradiction to the hypothesis that UV radiation is required for the production of the carriers (see Herbig 1995; Kendall et al. 2002, for example). However, MAG 63885 and MAG 70817 are near to the OB78 association where the abundance of early-type stars may result in a strong interstellar UV radiation field.

The M $312175 \AA$ UV extinction bump has been found to be weak and narrow (Bianchi et al. 1996; Hutchings et al. 1992) and a peculiar extinction law was observed by Massey et al. (1995) in the anomalously low average colour-excess ratio $E_{U-B} / E_{B-V}$. Accepting current theories of dust grain extinction (see review by Draine 2003), the evidence is consistent with a different distribution of dust grain sizes and compositions compared to the Milky Way average. In particular, there may be a lack of small graphitic dust grains (see also Xu \& Helou 1994). In that case, the observation of strong DIBs implies that the carriers are not closely associated with the small grains believed to be responsible for shape of the UV extinction curve.

\section{Conclusion}

The $\lambda \lambda 5780,5797,6283,6203$ and 6613 DIBs were detected towards MAG 63885 and MAG 70817 at velocities corresponding (within $25 \mathrm{~km} \mathrm{~s}^{-1}$ ) to the mean M 31 interstellar Na I absorption. $\lambda 6196$ and $\lambda 4430$ were also tentatively detected.

The M 31 DIB spectrum towards MAG 63885 is consistent with that observed in the Galaxy. Towards MAG 70817 the DIBs are about a factor of three stronger per unit reddening than the Galactic average. The high DIB strengths might be related to differences in the M31 interstellar UV extinction curve and radiation field compared to the Galaxy. Further studies will be required to determine if the two sightlines examined here are representative of the general M 31 DIB behaviour.

A planned instrument for the Hubble Space Telescope, the "Cosmic Origins Spectrograph" will be able to provide essential information on the UV properties of M 31 sightlines to further examine these possibilities. However, the connection between DIBs in M31 and the atomic and molecular content (e.g. Na, $\mathrm{K}, \mathrm{Ca}, \mathrm{CH}^{+}, \mathrm{CH}, \mathrm{CN}$ and $\mathrm{C}_{2}$ ) of the diffuse medium can only be properly addressed with high resolution, high signal-to-noise optical spectroscopic observations.

Acknowledgements. The W. M. Keck Observatory is operated as a scientific partnership among the California Institute of Technology, the University of California, and NASA. The observatory was made possible by the generous financial support of the W. M. Keck Foundation. The analysis pipeline used to reduce the Keck/DEIMOS data was developed at UC Berkeley with support from NSF grant AST-0071048. This research has made use of NASA's Astrophysics Data System. MAC and NLJC acknowledge support from the Faculty of the European Space Astronomy Centre (ESAC). MAC thanks QUB for financial support.

\section{References}

Bianchi, L., Clayton, G. C., Bohlin, R. C., Hutchings, J. B., \& Massey, P. 1996, ApJ, 471, 203

Bresolin, F., Kudritzki, R.-P., Lennon, D. J., et al. 2002, ApJ, 580, 213

Burstein, D., \& Heiles, C. 1978, ApJ, 225, 40

Cesarsky, D., Lequeux, J., Pagani, L., et al. 1998, A\&A, 337, L35

Cordiner, M. A. 2006, Ph.D. Thesis, The University of Nottingham

Cordiner, M. A., Cox, N. L. J., Trundle, C., et al. 2008, in preparation

Cox, N. L. J., Cordiner, M. A., Cami, J., et al. 2006, A\&A, 447, 991

Cox, N. L. J., Cordiner, M. A., Ehrenfreund, P., et al. 2007, A\&A, 470, 941

Draine, B. T. 2003, ApJ, 598, 1017

Ehrenfreund, P., \& Foing, B. H. 1996, A\&A, 307, L25

Ehrenfreund, P., Cami, J., Jiménez-Vicente, J., et al. 2002, ApJ, 576, L117

Ellison, S. L., York, B. A., Murphy, M. T., et al. 2008, MNRAS, 383, L30

Evans, C. J., \& Howarth, I. D. 2003, MNRAS, 345, 1223

Evans, C. J., Howarth, I. D., Irwin, M. J., Burnley, A. W., \& Harries, T. J. 2004, MNRAS, 353, 601

Galazutdinov, G. A., Musaev, F. A., Krełowski, J., \& Walker, G. A. H. 2000, PASP, 112, 648

Herbig, G. H. 1993, ApJ, 407, 142

Herbig, G. H. 1995, ARA\&A, 33, 19

Hobbs, L. M. 1974, ApJ, 191, 381

Howarth, I. D., Price, R. J., Crawford, I. A., \& Hawkins, I. 2002, MNRAS, 335, 267

Hutchings, J. B., Bianchi, L., Lamers, H. J. G. L. M., Massey, P., \& Morris, S. C. 1992, ApJ, 400, L35

Jenniskens, P., \& Desert, F.-X. 1994, A\&AS, 106, 39

Johnson, H. L. 1966, ARA\&A, 4, 193

Kalberla, P. M. W., Burton, W. B., Hartmann, D., et al. 2005, A\&A, 440, 775

Kendall, T. R., Mauron, N., McCombie, J., \& Sarre, P. J. 2002, A\&A, 387, 624

Magnier, E. A., Lewin, W. H. G., van Paradijs, J., et al. 1992, A\&AS, 96, 379

Massey, P., Armandroff, T. E., Pyke, R., Patel, K., \& Wilson, C. D. 1995, AJ, 110,2715

Massey, P., Olsen, K. A. G., Hodge, P. W., et al. 2006, AJ, 131, 2478

Megier, A., Krełowski, J., \& Weselak, T. 2005, MNRAS, 358, 563

Nedialkov, P., Berkhuijsen, E. M., Nieten, C., \& Haas, M. 2000, in Proc., 232, ed. E. M. Berkhuijsen, R. Beck, \& R. A. M. Walterbos, 85

Pagani, L., Lequeux, J., Cesarsky, D., et al. 1999, A\&A, 351, 447

Rich, R. M. 1987, AJ, 94, 651

Ruiterkamp, R., Cox, N. L. J., Spaans, M., et al. 2005, A\&A, 432, 515

Salama, F., Bakes, E. L. O., Allamandola, L. J., \& Tielens, A. G. G. M. 1996, ApJ, 458, 621

Sarre, P. J. 2006, J. Molec. Spectrosc., 238, 1

Sarre, P. J., Miles, J. R., Kerr, T. H., et al. 1995, MNRAS, 277, L41

Schlegel, D. J., Finkbeiner, D. P., \& Davis, M. 1998, ApJ, 500, 525

Snow, T. P. 2002, ApJ, 567, 407

Sollerman, J., Cox, N., Mattila, S., et al. 2005, A\&A, 429, 559

Thorburn, J. A., Hobbs, L. M., McCall, B. J., et al. 2003, ApJ, 584, 339

Trundle, C., Dufton, P. L., Lennon, D. J., Smartt, S. J., \& Urbaneja, M. A. 2002, A\&A, 395, 519

van den Bergh, S. 1964, ApJS, 9, 65

Walterbos, R. A. M., \& Braun, R. 1994, ApJ, 431, 156

Welty, D. E., Federman, S. R., Gredel, R., Thorburn, J. A., \& Lambert, D. L. 2006, ApJS, 165, 138

Xu, C., \& Helou, G. 1994, ApJ, 426, 109

York, B. A., Ellison, S. L., Lawton, B., et al. 2006, ApJ, 647, L29 